\title{
PLED-based MnSOD Mimetic
}

National Cancer Institute

\section{Source}

National Cancer Institute. PLED-based MnSOD Mimetic. NCI Thesaurus. Code C102747.

A derivative of pyridoxyl ethyldiamine (PLED) and mimetic of the human mitochondrial manganese superoxide dismutase (MnSOD), with antioxidant, metal chelating and potential chemoprotective activities. Upon administration, PLED-based MnSOD mimetic mimics MnSOD and scavenges oxygen free radicals such as superoxide anion, hydrogen peroxide, and hydroxyl radical, thereby preventing oxygen free radical damage to macromolecules such as DNA and minimizing oxygen free radical-related chemotoxicity in normal tissues. In addition, this agent is able to strongly bind to iron. 